Research Article

\title{
Some Striking Points of Laparoscopic Enucleation of Pancreatic Insulinoma
}

\author{
Ali Aktekin ${ }^{1}$, Seher Tanrikulu ${ }^{2}$, Abdullah Ramazan ${ }^{3}$, Aylin Gonultas ${ }^{4}$ and Can Saracoglu ${ }^{1}$ \\ ${ }^{1}$ Department of General Surgery, Giresun University, Turkey \\ ${ }^{2}$ Department of Internal Medicine, Division of Endocrinology and Metabolism, Haydarpasa Numune Education and \\ Research Hospital, Saglık Bilimleri University, Turkey \\ ${ }^{3}$ Department of Radiology, Haydarpasa Numune Education and Research Hospital, Saglık Bilimleri University, Turkey \\ ${ }^{4}$ Department of Pathology, Haydarpasa Numune Education and Research Hospital, Saglık Bilimleri University, Turkey
}

\begin{abstract}
Introduction: Insulinoma mostly located at the body and the tail of the pancreas and are ideal candidates for minimal invasive surgery. This article presents our patients with diagnosis of insulinoma and discusses strategic and technical aspects of their surgical managements.

Patients: All three patients were female 31, 49 and 66-years-old presented with the symptoms of hypoglycemia and laboratory of insulinoma. CT and MRI demonstrated solid lesions $11 \times 9 \mathrm{~mm}, 10-15 \mathrm{~mm}$, and $15 \mathrm{~mm}$ in size. We performed laparoscopic enucleation procedures in all three patients. Patients were discharged with improved clinical symptoms and normal blood glucose levels without any surgical complications.

Discussion: Laparoscopic enucleation of insulinoma has been performed only in half of the cases due to difficulty in localization of the tumor during the surgery and also the risk of pancreatic leakage. We performed laparoscopic enucleation procedures in all three patients. Even though one of them was located at surgically difficult site, with the help of radiologic images we could find precise localization. Moreover, having experiences on pancreatic and laparoscopic surgery, recognizing their distinct nodularity and feeling the texture of tumor help surgeon to perform laparoscopic enucleation of tumor. One of them was located at posterior to body of pancreas, nearby the SMV and splenic vein, and their confluence. Despite of its close proximity to important structures, this tumor was successfully enucleated by laparoscopy. We demonstrated possibility, feasibility and safety of laparoscopic enucleation of insulinoma even it locates at difficult anatomical sites.
\end{abstract}

Keywords

Insulinoma, Enucleation, Laparoscopy

\section{Introduction}

Pancreatic neuroendocrine (PNET) tumors are originated from islet cells of pancreas and also known as islet cells tumors. They are a rare form of endocrine neoplasms and reported in 1 to $4 \%$ of all pancreatic tumors [1,2]. Their diagnosis rate has increased due to developments in imaging and histopathologic methods $[3,4]$. PNETs can be a functional and non-functional tumor. The most common functional PNET is sporadic insulinoma, accounting for $70-80 \%$ of the cases. Its incidence has been reported as 3-10 cases per million people per year $[5,6]$. Insulinomas are insulin-secreting tumors and are located almost exclusively in the pancreas especially in the body and the tail of the pancreas. Most of them are benign and solitary [7].

Insulinoma causes a distinct syndrome characterized by symptoms due to hypoglycemia. The symptoms are head- aches, confusion, visual disturbances, sweating, tremor, palpitations and typically associated with fasting due to central nervous system effects of hypoglycemia or catecholamine excess secondary to hypoglycemia. Clinical symptoms of hypoglycemia occur early in the course of the disease make them

*Corresponding author: Ali Aktekin, MD, Associated Professor, Surgeon, Department of General Surgery. Giresun University, Medical Faculty, Giresun, Post code: 28100, Turkey, Tel: +90532-598-72-07

Received: May 07, 2020

Accepted: June 18, 2020

Published online: June 20, 2020

Citation: Aktekin A, Tanrikulu S, Ramazan A, et al. (2020) Some Striking Points of Laparoscopic Enucleation of Pancreatic Insulinoma. J Surgical Endocrinol 2(1):26-29 
small in size at the time of diagnosis and also suitable for surgery [5]. Surgical treatment options include tumor enucleation or regional pancreatic resection [8]. The small, benign, and solitary natures make insulinoma ideal candidates for laparoscopic and robotic approaches. First use of laparoscopy in resection of PNETs was reported by Gagner, et al. in 1996 [9].

This article presents our patients with diagnosis of insulinoma and discusses both the strategic and technical aspects of their surgical managements.

\section{Patients}

\section{Case one}

A 31-years-old female patient presented with the symptoms of the headaches, confusion, visual disturbances, sweating, tremor, palpitations after fasting. She reported that these symptoms were relieving after sugar based meal. Laboratory investigations with the suspicion of insulinoma had result in insulin levels of $11.5 \mu \mathrm{U} / \mathrm{mL}$, glucose $45 \mathrm{mg} / \mathrm{dL}$, and C-peptide $2.49 \mathrm{ng} / \mathrm{mL}$. The diagnosis of insulinoma was established by demonstrating inappropriately high serum insulin concentrations during hypoglycemia. Further evaluation of patient for MEN syndrome revealed pituitary adenoma and she was further evaluated for multiple endocrine neoplasm type 1.

The radiologic modalities we used to evaluate all patients were contrast enhanced computed abdominal tomography and dynamic magnetic resonance imaging. Moreover, we performed contrast enhanced three phase abdominal computed tomography, magnetic resonance cholangiopancreatography and endosonography, if they were available or needed at that time, to show and document precise localization and size of the tumor, its anatomic relation to superior mesenteric and splenic vessels, and also the pancreatic duct. We also evaluated the multiple or malignant PNET with these radiological modalities.

Tri-phasic contrast enhanced computed tomography of abdomen revealed a solid $11 \times 9 \mathrm{~mm}$ lesion in close proximity

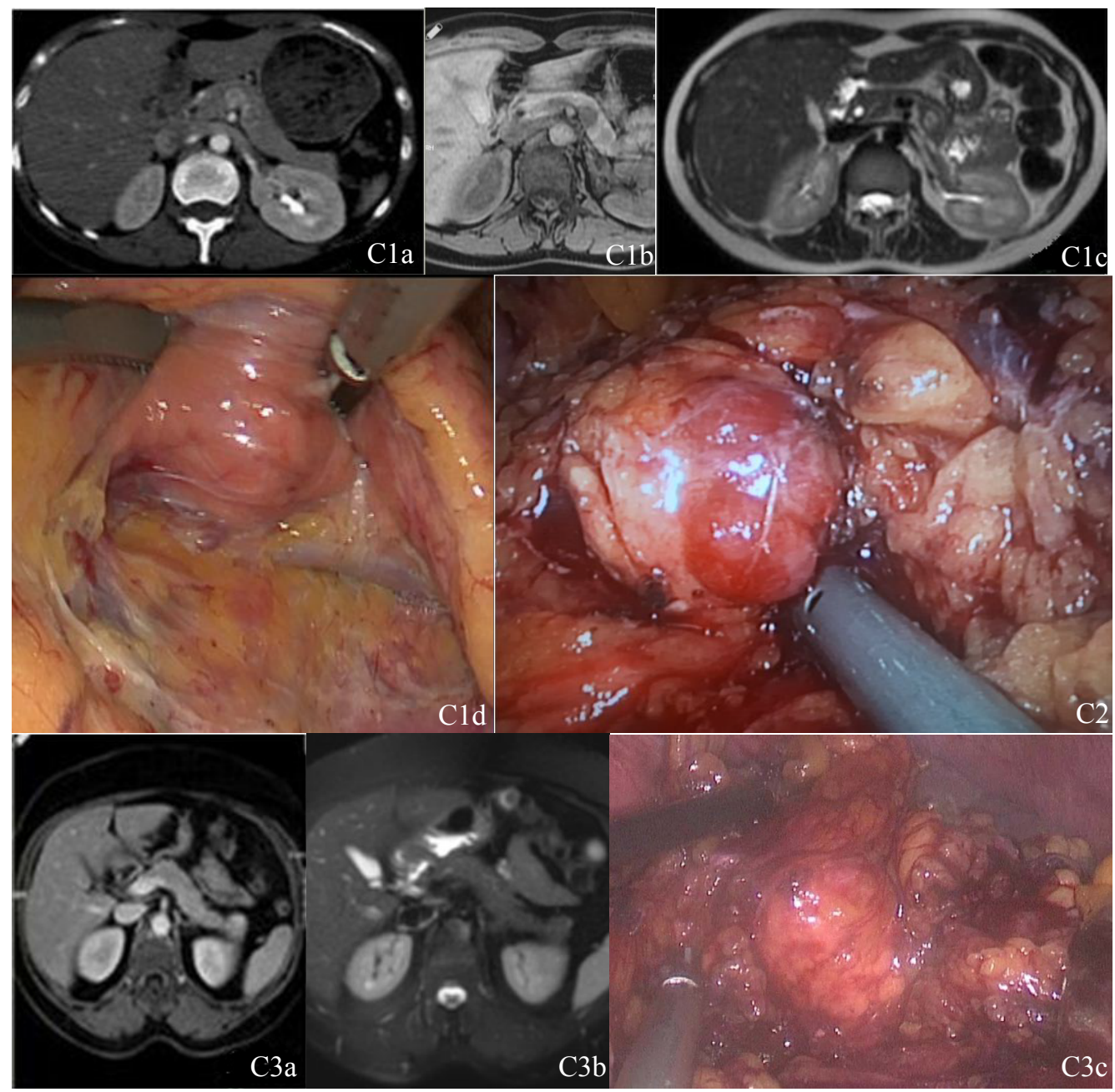

Figure 1: Radiologic images and laparoscopic views of patients operated for insulinoma. C1a, C1b, and C1c are the radiologic images of the first patient and demonstrate a solid lesion at close proximity to SMV and splenic vein and their confluence posterior to body of pancreas. C1d is a view of surgical field during laparoscopic surgery and shows a nodular lesion nearby to SMV and splenic vein and their confluence posterior to body of pancreas. C2 is an operative view of second patient demonstrating distinct nodularity of the tumor. C3a and $\mathbf{C 3 \mathbf { b }}$ are the radiologic images of third patient and demonstrate a lobulated solid lesion with close relation to splenic vein on anterior surface of pancreatic tail. C3c is the operative view of third patient with a distinct nodularity of tumor. 
to SMV and splenic vein confluence posterior to the body of pancreas. Dynamic contrast enhanced MRI demonstrated a lobulated solid $13 \times 15 \mathrm{~mm}$ lesion and shown isointensity with pancreas at T1 and T2-weighted images, but after contrast injection, it became a contrast enhanced lesion at arterial and late phases. Its localization was compatible with the location in computed tomography. Magnetic resonance cholangiopancreatography was performed to evaluate the relationship of the tumor with pancreatic duct. No relation with pancreatic duct was demonstrated (Figure $1 \mathrm{C} 1 \mathrm{a}$, Figure $1 \mathrm{C} 1 \mathrm{~b}$ and Figure 1C1c).

\section{Case two}

A 49-years-old female patient presented with the symptoms hypoglycemia after fasting and relieving these symptoms after sugar based meal. Laboratory evaluation revealed insulin level $35.4 \mu \mathrm{U} / \mathrm{mL}$, glucose $43 \mathrm{mg} / \mathrm{dL}$ after fasting period and C-peptide level $3.15 \mathrm{ng} / \mathrm{mL}$. Contrast enhanced computed tomography of abdomen demonstrated a hyperdense 13 $\mathrm{mm}$ lesion at the tail of pancreas. Dynamic contrast enhanced MRI revealed a $10 \mathrm{~mm}$ solid hypointense lesion at T1-weighted scans, similar intensity with pancreas at T2-weighted scans but after contrast injection, tumor has not lose signals even at the portal phase. Linear EUS revealed a solid $15 \mathrm{~mm}$ lesion at the tail of pancreas. All these radiologic modalities revealed no relationship of the tumor with pancreatic duct.

\section{Case three}

A 66-years-old female patient presented with the symptoms hypoglycemia after fasting and relieving these symptoms after sugar based meal. Laboratory evaluation revealed insulin level $10.8 \mu \mathrm{U} / \mathrm{mL}$, glucose $40 \mathrm{mg} / \mathrm{dL}$ after supervised fasting period with C-peptide $1.87 \mathrm{ng} / \mathrm{mL}$. Further evaluation of patients for MEN syndrome revealed pituitary adenoma and evaluated for multiple endocrine neoplasm type 1 . Although contrast enhanced computed tomography of abdomen revealed no pathology, dynamic contrast enhanced MRI revealed a lobulated $15 \mathrm{~mm}$ solid lesion which shown isointensity with pancreas at T1 and T2-weighted scans, but after contrast injections, it became a highly contrast enhanced at both arterial and late phases. It had close proximity to splenic vein on anterior surface of pancreatic tail. Radiologic modalities demonstrated no relationship of the tumor with pancreatic duct (Figure $1 \mathrm{C3a}$ and Figure 1C3b).

\section{Surgical procedures}

After insufflations of the abdomen, gastrocolic and gastrosplenic ligaments were open with the preserving of gastroepiploic vessels and approached to lesser omental sac. Up to location of the tumor, inferior border of pancreas dissected from retroperitoneal attachments. Tumor was investigated at pancreas on its assumed localization with the aids of its preoperative radiological images, its distinct nodularity, and their slightly different colour from the back ground of pancreatic tissue. I could also feel the some tactile differences of the tumors from other normal pancreatic tissues through the laparoscopic instruments. They had rough texture than normal pancreatic tissue.
We found all the tumors at exactly the same locations described by radiological evaluations. Tumor of the first patient was located nearby to both superior mesenteric and splenic veins and their confluence at posterior to body of the pancreas (Figure $1 \mathrm{C} 1 \mathrm{~d}$ ). The tumor of second patient was found at inferior border of the distal pancreatic tail (Figure 1C2). The tumor of third patient was found on anterior surface of the pancreatic tail and closely proximal to splenic vein (Figure 1C3c).

With the ultrasonic dissector, tumors were enucleated with a thin rim of pancreas to minimize the risk of fragmentation of tumors. After tumors were taken out of abdomen within an endoscopic bag, frozen sections were evaluated to verify that lesion was a PNET and also ensure clear margins of the tumor. Tumor sites were irrigated and cleaned well with saline solution. Careful observation was done to check integrity of pancreatic duct and to observe any leakage from pancreas. Some tissue adhesive materials were put on enucleated sites to prevent leakage if any missed pancreatic duct injury was present [10]. Non-suctioning closed system drains were put near the wound of the enucleated sites and operations were ended.

\section{Clinical and laboratory evaluations after surgery}

All the patients were evaluated for clinical symptoms of hypoglycemia and blood glucose levels were closely monitored. We preferred to delay the withdrawal of drains after discharging from hospital to ensure no pancreatic leakage present. We did not encounter any pancreatic leakages and all symptoms of patients were revealed.

\section{Histopathologic analysis}

Histopathological evaluations of tumors were reported as PNET in all of the patients. Their sizes were $20 \times 20 \times 15,20 \times$ $15 \times 15$ and $12 \times 10 \times 10 \mathrm{~mm}$, in order of case presentation, with a thin rim of normal pancreatic tissue or some capsules. Immunohistochemical staining of the all tumors was reported chromogranin and synaptophysin and insulin, if available, positivity; histochemical analysis revealed congo red stained tissues with abandoned amyloidal deposits. All of three cases were diagnosed as well differentiated PNET.

\section{Discussion}

Surgery is the treatment of choice for insulinoma and cures in over $90 \%$ of patients. If surgery is not mandible, interventional ablative procedures or medical treatments can be used to control of insulin over secretion [11]. As most of the insulinomas are in benign nature, enucleation is the preferred and recommended procedure to preserve pancreas, but unfortunately, nowadays enucleation performed only half of the cases due to the difficulty in localization of the tumor during the surgery and also the risk of pancreatic leakage $[12,13]$. On the other hand, it should be kept in mind that enucleation prevents the risk of pancreas insufficiency [14].

With the introduction of laparoscopic resection, patients have shorter postoperative stay and pain without increased prevalence of pancreatic fistula and recurrence rate or postoperative mortality $[12,15,16]$. Many studies have recommend- 
ed laparoscopic enucleation of insulinoma as the treatment of choice in patients with sporadic, single and benign tumors because of its feasibility and safety [12,17]. Even Al-Kurd, et al. published a review article at 2014 and concluded that the rate of major complications is higher in those undergoing open surgery than minimal invasive surgery [17]. However, the open approach remains the most common method for insulinoma enucleation due to lack of digital palpation during laparoscopic enucleation of the tumor. Performing intraoperative laparoscopic ultrasonography would help to identify the tumor during laparoscopic and also robotic surgery [18-20].

We performed laparoscopic procedures in all three patients. We evaluated location and proximity of tumor to adjacent structures like SMV, splenic vein, and especially to pancreatic duct by preoperative radiological studies. These imaging technics also help us to detect multifocal and malignant tumors. On the other hand, we could feel the rough texture of tumor from normal pancreatic tissue with laparoscopic instruments. They also had distinct nodularity that helped us to localize the tumors. In addition we are very experienced on laparoscopic procedures and perform laparoscopic obesity, adrenal and splenic procedures and also open pancreatic surgery that help us to precisely identify anatomic structures during laparoscopic surgery. Although Belfiori, et al. recommended that all benign sporadic insulinoma localized ventral in the head and body should be approached with a laparoscopic or robotic enucleation intention, we also successfully performed laparocopic enucleation of an insulinoma in 20 $\mathrm{mm}$ size and closely proximal to superior mesenteric and splenic vein and their confluence at posterior to body of the pancreas [20].

The results presented in these studies demonstrate the feasibility and safety of laparoscopic enucleation of insulinoma in experienced hands with complete cure of patients without postoperative complications. Careful evaluation of the radiological images before operation and having experiences on laparoscopic pancreatic surgery are the important points for laparoscopic enucleation of insulinoma. The distinct nodularity and texture of the tumor, some tactile feeling through the laparoscopic instruments also help to localize the tumor.

\section{References}

1. Eriksson B, Oberg K (2000) Neuroendocrine tumours of the pancreas. Br J Surg 87: 129-131.

2. Fendrich V, Bartsch DK (2011) Surgical treatment of gastrointestinal neuroendocrine tumors. Langenbecks Arch Surg 396: 299-311.

3. Yao JC, Hassan M, Phan A, et al. (2008) One hundred years after "carcinoid": Epidemiology of and prognostic factors for neuroendocrine tumors in 35,825 cases in the United States. J Clin Oncol 26: 3063-3072.

4. Halfdanarson TR, Rabe KG, Rubin J, et al. (2008) Pancreatic neuroendocrine tumors (PNETs): Incidence, prognosis and recent trend toward improved survival. Ann Oncol 19: 1727-1733.

5. Fernández-Cruz L, Herrera M, Sáenz A, et al. (2001) Laparoscop- ic pancreatic surgery in patients with neuroendocrine tumours: Indications and limits. Best Pract Res Clin Endocrinol Metab 15: 161-175.

6. Anderson CW, Bennett JJ (2016) Clinical presentation and diagnosis of pancreatic neuroendocrine tumors. Surg Oncol Clin N Am 25: 363-374.

7. Guettier JM, Gorden P (2010) Insulin secretion and insulin-producing tumors. Expert Rev Endocrinol Metab 5: 217-227.

8. Jensen RT, Cadiot G, Brandi ML, et al. (2012) ENETS Consensus Guidelines for the management of patients with digestive neuroendocrine neoplasms: Functional pancreatic endocrine tumor syndromes. Neuroendocrinology 95: 98-119.

9. Gagner M, Pomp A, Herrera MF (1996) Early experience with laparoscopic resections of islet cell tumors. Surgery 120: 10511054.

10. Conzo G, Gambardella C, Tartaglia E, et al. (2015) Pancreatic fistula following pancreatoduodenectomy. Evaluation of different surgical approaches in the management of pancreatic stump. Literature review. International Journal of Surgery 21: S4-S9.

11. Mele C, Brunani A, Damascelli B, et al. (2018) Non-surgical ablative therapies for inoperable benign insulinoma. J Endocrinol Investig 41: 153-162.

12. M Falconi, B Eriksson, G Kaltsas, et al. (2016) Consensus guidelines update for the management of functional p-NETs (F-pNETs) and non-functional p-NETs (NF-p-NETs). Neuroendocrinology 103: 153-171.

13. Wolk S, Distler M, Kersting S, et al. (2015) Evaluation of central pancreatectomy and pancreatic enucleation as pancreatic resections-a comparison. Int J Surg 22: 118-124.

14. Bartsch DK, Albers M, Knoop R, et al. (2013) Enucleation and limited pancreatic resection provide long-term cure for insulinoma in multiple endocrine neoplasia type 1. Neuroendocrinology 98: 290-298.

15. Tamburrino D, Partelli S, Renzi C, et al. (2017) Systematic review and meta-analysis on laparoscopic pancreatic resections for neuroendocrine neoplasms (PNENs). Expert Rev Gastroenterol Hepatol 11: 65-73.

16. Su AP, Ke NW, Zhang Y, et al. (2014) Is laparoscopic approach for pancreatic insulinomas safe? Results of a systematic review and meta-analysis. J Surg Res 186: 126-134.

17. Al-Kurd A, Chapchay K, Grozinsky-Glasberg S, et al. (2014) Laparoscopic resection of pancreatic neuroendocrine tumors World J Gastroenterol 20: 4908-4916.

18. Han X, Wu W, Wang M, et al. (2016) Combination of intraoperative ultrasonography for localizing insulinoma under Da Vinci robotic surgical system: Experience of a single center in 50 cases. Zhonghua Wai Ke Za Zhi 54: 30-33.

19. Dalvi AN, Garale MN, Takalkar YP, et al. (2018) Laparoscopic surgery for solitary insulinoma in the absence of IOUS. J Minim Access Surg 14: 9-12.

20. Belfiori G, Wiese D, Partelli S, et al. (2018) Minimally invasive versus open treatment for benign sporadic insulinoma comparison of short-term and long-term outcomes. World J Surg 42: 3223-3230.

Copyright: (C) 2020 Aktekin A, et al. This is an open-access article distributed under the terms of the Creative Commons Attribution License, which permits unrestricted use, distribution, and reproduction in any medium, provided the original author and source are credited. 